\title{
DIEZ NOTAS LEXICOGRÁFICAS A PÍNDARO ${ }^{1}$
}

\author{
Helena Rodríguez Somolinos \\ ILC - CSIC \\ helena.rsomolinos@cchs.csic.es
}

\section{RESUMEN}

Reunimos aquí diez notas lexicográficas sobre palabras procedentes de los textos del poeta Píndaro, que formarán parte de la próxima entrega del Diccionario Griego-Español. Todas ellas aportan alguna novedad con respecto a los diccionarios generales anteriores o con respecto a la interpretación habitual del término o de su contexto.

Palabras Clave: Léxico. Píndaro. Diccionario Griego-Español.

\section{Abstract}

Here we gather ten lexicographical notes about words coming from the poet Pindar's texts, which will be shown in the next Greek-Spanish Dictionary volume. All of them provide some novelties in relation with the previous general dictionaries or with the interpretations and readings that are usually accepted.

KEY WORDS: Vocabulary. Pindar. Greek-English Dictionary.

${ }^{1}$ Es una gran satisfacción poder dedicarle estas notas a José María Lucas, compañero y amigo, a quien tanto deben Epos, el Departamento de Filología Clásica de la UNED y los estudios clásicos en España, y que tan estrecha relación ha tenido con el Diccionario Griego-Español. Este trabajo ha sido elaborado dentro del Proyecto de Investigación FI2010-20236 «Diccionario Griego-Español: continuación» financiado por el Plan Nacional I+D+i. 
Por la complejidad sintáctica del texto de Píndaro y por la frecuente oscuridad conceptual que suele acompañarlo, el vocabulario procedente de sus poemas presenta, en general, escaso valor desde el punto de vista lexicográfico, al margen del enorme valor que otorgan a su testimonio la importancia del autor y su alta fecha. El trabajo de redacción en un diccionario como el Diccionario Griego-Español (DGE) permite darse cuenta de que, con Píndaro, a menudo es difícil ofrecer un contexto breve que se explique por sí solo y que ayude a entender el significado de la palabra en cuestión, si no se acompaña de información complementaria que aclare el contexto amplio en el que aparece la palabra y los referentes y alusiones que hay detrás de las frecuentes metáforas e imágenes. Por las mismas razones, a menudo se plantean numerosas dificultades en la interpretación de pasajes o palabras que, ya más allá de la labor estrictamente lexicográfica, hacen imprescindible la consulta de bibliografía especializada, muy en particular de los excelentes comentarios que han aparecido en las últimas décadas.

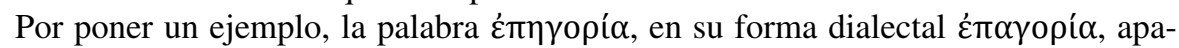
rece en dos fragmentos de Píndaro, lo que constituye en sí un material interesante por ser de Píndaro y porque, aparte de una cita de Dión Casio (55.18), no hay otra documentación del significado acusación, reproche ${ }^{2}$. El Fr.359 consiste únicamente en las


aunque lo expongamos con mayor amplitud de la apropiada para un diccionario, no aporta mucho al sentido de la palabra, ni siquiera a la comprensión del propio contexto:


$\delta \rho \varepsilon ́ \pi \varepsilon \sigma \theta \alpha$, en lo que sería probablemente la forma mínima de este contexto. Pero para que este contexto aporte claridad al significado de la palabra es imprescindible saber que el poeta se está dirigiendo a las prostitutas sagradas del Acrocorinto, a las que Afrodita concedió cosechar en blandos lechos el fruto de su tierna juventud, es decir, mantener relaciones sexuales sin reproche, porque es un acto religioso y es su obligación. Por eso

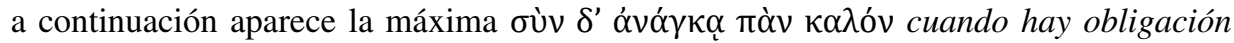
todo está bien. Resulta imposible introducir tanta explicación, razón por la cual en el

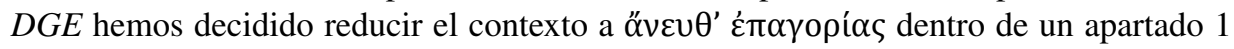

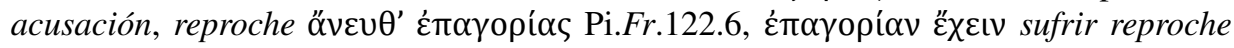
Pi.Fr.359, D.C.55.18.

Es Píndaro, pues, un autor especialmente difícil de entender, y la información léxica que proporciona precisa de un manejo especialmente delicado. Aquí reunimos unas pocas notas relativas a palabras que aparecerán recogidas en el volumen VIII del Diccionario Griego-Español, y surgidas en concreto de la revisión sistemática de las citas de cada autor que constituye una de las partes más importantes de la fase de revisión del original del $D G E$. Ofrecemos en general la información más relevante, sin detenernos en el estudio pormenorizado de la bibliografía sobre cada pasaje, y ejemplificando las traducciones modernas en las tres más habituales al castellano, las de Ortega (1984), Bádenas-Bernabé (1984) y Suárez de la Torre (1988).

${ }^{2}$ La fuente del fragmento, Chamael.37 en Ath.13.573fss. transmite $\alpha ́ \pi \alpha \gamma o \rho i ́ \alpha$. En LSJ el testimonio de Píndaro s.u. Éтпү $\gamma$ opí $\alpha$ aparece como conjetura, sin que quede claro qué edición sigue; en la edición de referencia del $D G E$, la de Maehler (1989) en Teubner, la conjetura es aceptada en el texto, como es aceptada por Giordano en su edición de Cameleonte (Giordano 1990²). 
En todas ellas aportamos alguna novedad con respecto a nuestro inmediato predecesor, el diccionario de Liddell-Scott-Jones, o con respecto a la interpretación habitual del término o de su contexto. En algunos casos ha sido el estudio minucioso de la documentación de la palabra en su conjunto lo que ha permitido mejorar la interpretación de pasaje pindárico. En otros la clave está en una mejor comprensión de los preverbios, ya que la sección de vocabulario comprendida en el volumen VIII del DGE consiste en un veinte por ciento en compuestos de $\varepsilon^{\xi} \xi$-, y en casi un setenta por ciento en compuestos de $\dot{\varepsilon} \pi(\mathrm{l})-$. En particular, creemos que una de las principales aportaciones de este volumen está en la comprensión de los significados y funciones del preverbio ćđt-, frecuentemente ignorado o malinterpretado en los diccionarios y traducciones. En otros casos encontramos un significado especial en LSJ que, estudiado en profundidad, resulta ser tan sólo un uso figurado o metafórico esporádico donde lo oportuno precisamente es mantener la traducción propia, para no eliminar la metáfora o la imagen. En alguna ocasión, finalmente, aportamos algún addendum procedente de la bibliografía reciente o justificamos la inclusión de información textual, cuando es relevante.

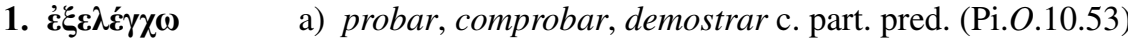 \\ b) probar la realidad de algo, verificar (Pi.N.10.46)}

En LSJ s.u. la primera de estas dos citas de Píndaro encabeza un apartado II put

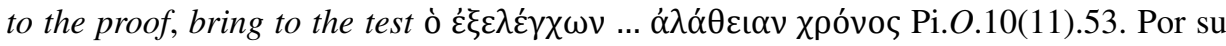
parte, Slater la entiende como prove, be the proof of.

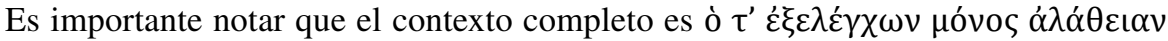


vo. Así lo hacen, sin salir de las traducciones castellanas más habituales, bastante libres — salvo la última - en cuanto al significado del verbo: «y el único garante de la auténtica verdad, el Tiempo» (Bádenas - Bernabé), «y el único que da testimonio de la verdad real» (Ortega), «y el único que demuestra la verdad auténtica, el Tiempo» (Suárez).

La comprensión de los distintos significados de $\varepsilon^{\lambda} \varepsilon^{\gamma} \gamma \chi \omega$ y sus compuestos se ve a menudo dificultada por la ambivalencia de sus equivalentes más inmediatos en las lenguas modernas. En este caso es fundamental diferenciar entre lo que es probar como proceso (someter a prueba a alguien o algo) y lo que es probar como comprobar, demostrar, es decir, alcanzar un resultado al término de dicho proceso.

$D G E$ ha organizado los sentidos generales o neutros del verbo (A por oposición a $\mathbf{B}$ «en sent. neg.») es decir, sin noción negativa, a partir de una primera división entre ambas nociones. Hay así un apartado I, en donde el verbo indica un proceso (probar, someter a prueba) y el complemento será un acusativo (la poesía de Esquilo y Eurípides para ver

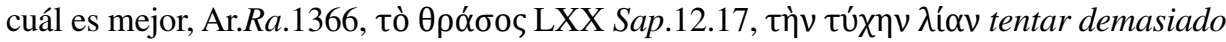
a la suerte Plb.21.14.4, etc.), y un apartado II en el que el verbo incide en el resultado (probar, comprobar, demostrar un hecho o idea) y generalmente (subapartado 1) va


$\dot{\varepsilon} \xi \dot{\eta} \lambda \varepsilon \gamma \xi \alpha v$ probaron que los indios no tenían oro), o bien de una oración completiva (Arr.

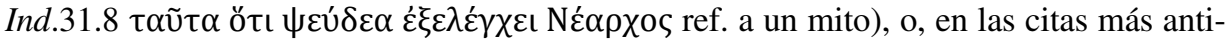
guas, de una construcción con participio predicativo. Así, en voz activa en Pl.Grg.522d عí




soy incapaz de ayudarme a mí mismo y a otro», y en voz pasiva con participio concertado

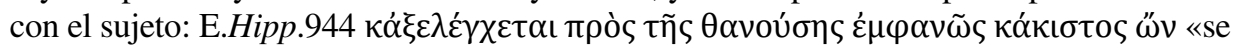
demuestra claramente con el testimonio de la muerta que es el más malvado».

Estos dos pasajes en que el verbo se construye con un participio, y en particular el uso activa de Platón, nos dan la clave para entender la cita de Píndaro, donde claramente

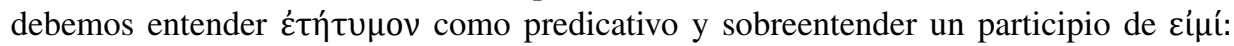

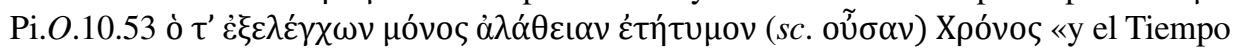
que es el único que demuestra que la verdad es auténtica».

En el segundo pasaje (Pi.N.10.45s.), Píndaro emite una afirmación general:

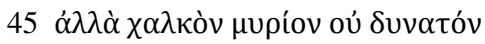

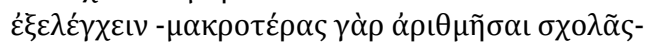

En LSJ ésta es la única cita de un apartado III compute $\chi \alpha \lambda$ кòv $\mu$ píov Pi.N.10.46. Así pues, hay que entender la frase como «pero no es posible contar innumerable bronce» lo cual es una traducción sumamente libre tomada de identificar directamente $\dot{\varepsilon} \xi \varepsilon \lambda \varepsilon \dot{\varepsilon} \gamma \chi \varepsilon \iota \nu$ con $\alpha \rho \imath \theta \mu \tilde{\eta} \sigma \alpha \iota$, y demasiado contextual como para dar lugar a un apartado con número romano por sí sola. Más acertado está Slater, que lo entiende como ascertain, es decir, verificar, comprobar. Este significado entraría en el mencionado apartado II, con la salvedad respecto a los ejemplos que hemos visto de que el complemento es un acusativo. En este caso, por tanto, se trataría de probar, comprobar la realidad de algo, comprobar que algo es cierto, es decir, verificar.

Efectivamente dentro de dicho apartado II, $D G E$ ha hecho un subapartado para este caso (II 2 c. ac. de cosa probar la realidad de algo, verificar, contrastar), en el que aparece una cita de Menandro en voz pasiva que podría considerarse cercana: en v. pas.

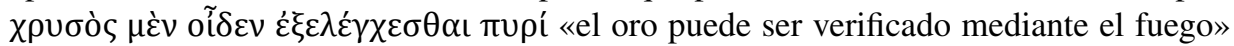
Men.Comp.2.83.

La dificultad del testimonio pindárico estriba en que podemos entender la acción verbal en los dos sentidos, como proceso o como fin del proceso. Si consideramos que

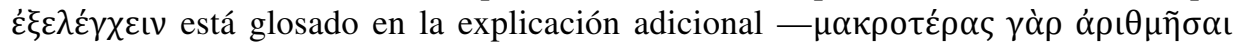
$\sigma \chi 0 \lambda \tilde{\alpha} \varsigma$ - entonces el verbo implicaría un proceso, el largo proceso de someter a examen o comprobación los trofeos deportivos, es decir, las victorias previas, mediante su recuento ( $\alpha \rho \imath \theta \mu \tilde{\eta} \sigma \alpha \iota)$. En tal caso esta cita debería ir unida a Plb.1.62.4, donde Hamílcar

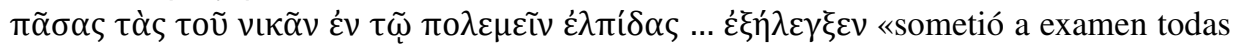
las oportunidades de vencer en la guerra».

Sin embargo, hemos considerado mejor incluir la cita en el mencionado apartado

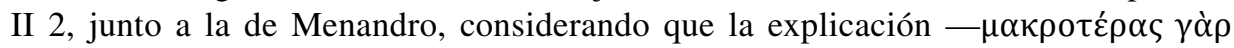

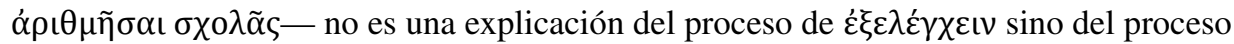
previo que habría que seguir para alcanzar la demostración. Es decir, Píndaro estaría diciendo que no es posible demostrar la realidad de tanto bronce, verificarlo pues para ello habría que hacer un cómputo previo que requeriría demasiado tiempo.

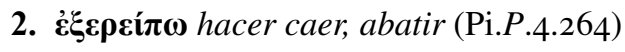

En LSJ el lema $\varepsilon \dot{\xi} \varepsilon \rho \varepsilon i ́ \pi \omega$ presenta un primer apartado transitivo para esta cita

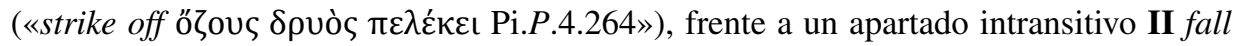


to earth donde se agrupan diversas citas de Homero, Hesíodo e Hipócrates. Slater ofrece la misma traducción strike off añadiendo amplia información textual que no afecta a la existencia misma del verbo, aunque sí a su forma.

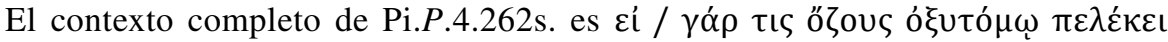
$\varepsilon \dot{\xi} \xi \rho \varepsilon i ́\{\varepsilon เ \varepsilon \nu \mu \varepsilon \gamma \alpha \dot{\alpha} \lambda \alpha \varsigma \delta \rho$ ós, en versión de Snell-Maehler que también aceptan la con-

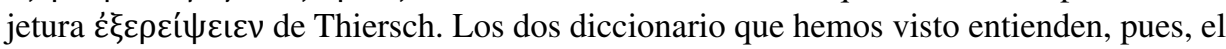
verbo como cortar, cercenar, quitar de golpe. Los traductores modernos suelen inclinarse por cortar, talar, podar y sus equivalentes en otras lenguas.

Este caso constituye un excelente ejemplo de un error de traducción bastante común, que consiste en traducir un verbo no por lo que significa realmente sino por otro verbo que expresa lo que está pasando. Naturalmente, un traductor puede permitírselo, pero un lexicógrafo no puede caer en este error. Quizá haya ayudado a ello la excepcional circunstancia de que aquí el verbo $\varepsilon_{\xi}^{\varepsilon} \rho \varepsilon \varepsilon^{\prime} \pi \omega$ es transitivo. Es claro que si alguien emplea el hacha contra las ramas de un árbol es para cortarlas, pero el uso transitivo de este verbo, que normalmente significa caer a tierra, desplomarse debe entenderse como factitivo: hacer caer, abatir, derribar las ramas.



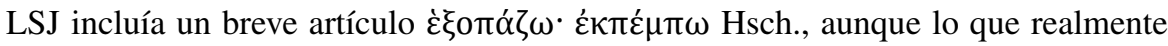

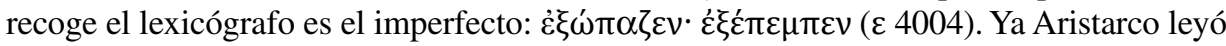
la $3^{\text {a }}$ pers. sg. del aoristo de este verbo en Pi.I.1.11, donde los editores, incluidos SnellMaehler, suelen leer el verbo simple precedido del numeral ह̌ $\xi$ :



Esas supuestas seis victorias previas de los tebanos en los juegos del Istmo han dado lugar a abundante bibliografía desde los propios escolios a Píndaro. En 1982 Aurelio Privitera retomó esta lectura, defendiéndola de forma muy convincente, por lo que el $D G E$ ha optado por incluir la cita. En cuanto al significado, aceptamos, con Privitera, escasa diferencia entre el verbo compuesto y el simple. El artículo resultante en $D G E$ es:

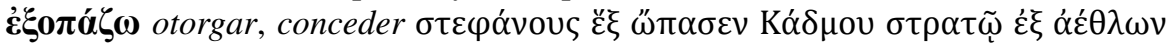
Pi.I.1.11 (var.), cf. Hsch.ع 4004.



LSJ establece tres significados, agrupados dos de ellos en un apartado A como significados propios y reservando para el tercero, metafórico, un apartado especial, que es donde aparecen las dos citas de Píndaro. Resumimos el primero y reproducimos los dos segundos tal y como aparecen en el diccionario inglés:

I 1 weave $\varphi \tilde{\alpha} \rho \circ$ Sdt.2.11, etc. 



Gp.15.5.2.

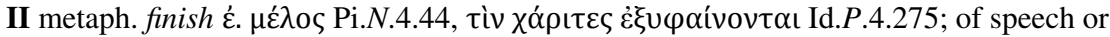

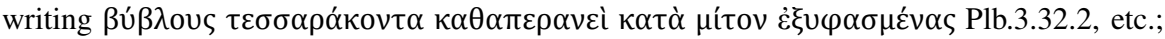

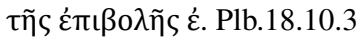

Hay un problema de base en la interpretación de este verbo, pues si se estudia a

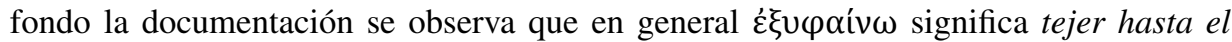
final, terminar de tejer, no sólo en las dos citas del apartado I 2 de LSJ, que es donde este significado resulta más evidente. Por eso el complemento directo suele ser de resultado, desde Hdt.2.122 $\varphi \tilde{\alpha} \rho \circ \varsigma \delta \grave{\varepsilon} \alpha u ́ \tau \eta \mu \varepsilon \rho o ̀ v ~ \dot{\varepsilon} \xi \cup \varphi \eta ́ v \alpha \nu \tau \varepsilon \varsigma$ «terminando de tejer una tela en el mismo día», donde el adverbio también nos indica el fin del proceso.

Al margen de ello, en lo que se refiere a las citas metafóricas agrupadas en el apartado II, encontramos un problema habitual en la organización de artículos de diccionario, que es saber cuándo los usos figurados y metafóricos deben ir integrados en el apartado de significados propios y cuándo deben disponer de un apartado especial. Como norma general, en $D G E$ entendemos que si la traducción no cambia deben aparecer junto a los usos propios, indicando la peculiaridad de que se trata de un uso figurado. El problema en este caso es similar al que hemos visto en $\grave{\varepsilon} \xi \varepsilon \rho \varepsilon i ́ \pi \omega$, y consiste en que se ha utilizado una traducción especial que describe la acción real, pero no la acción figurada, que es lo que quiere expresar el poeta. Ya estemos hablando de una tela o de un canto, como en

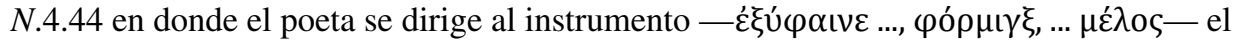
significado es el mismo, tejer hasta el final, y así debemos traducirlo, porque si no destruimos la metáfora y traicionamos al poeta. Sabemos de lo que Píndaro está hablando, de construir o ejecutar un poema hasta el final, pero no es eso lo que está diciendo. Y exactamente lo mismo sucede en los ejemplos de Polibio referidos al discurso hablado o escrito, a los que se han sumado otros ejemplos en el DGE. Por eso, siguiendo sus convenciones formales habituales, esta cita de Píndaro irá en $D G E$ unida a los usos propios, y precedida simplemente de un punto y coma y la indicación: fig. del poema o el discur-

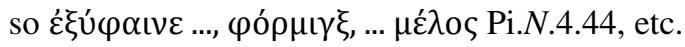

Más allá del contexto literario, la cita de Pi.P.4.275 nos habla de favores de los

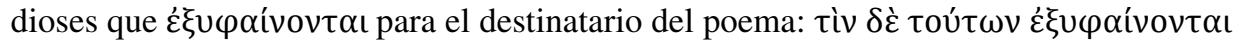

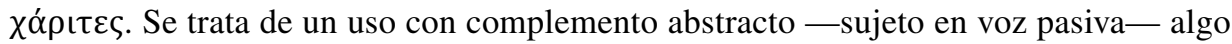
diferente, que podemos comparar, en primer término, con ejemplos del simple ù $\varphi \alpha i ́ v \omega$,

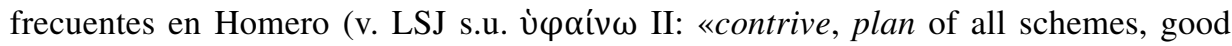
or bad, which are craftily imagined»). Pero hay diferencia en Píndaro con respecto a los usos figurados de v่ $\varphi \alpha$ ív $\omega$ en Homero. En éstos el verbo tiene matiz negativo y el complemento suele ser de resultado ( $\delta$ ó $\lambda$ ov, $\mu \tilde{\eta} \tau \iota v, \mu \tilde{v} \theta o \varsigma$, etc.). Normalmente traduciremos el verbo por urdir, tramar o directamente, más allá de metáforas, por planear, elaborar. Un ejemplo paralelo del compuesto sería el mencionado de Plb.18.10.3

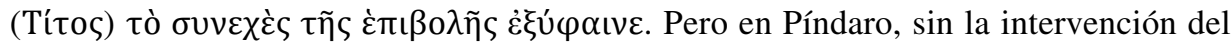
matiz negativo, la traducción adecuada sigue siendo tejer, como en el ejemplo más cercano del simple, que encontramos en la misma Pítica de Píndaro: Pi.P.4.141 $\alpha \lambda \lambda^{\prime}$

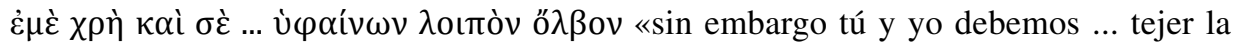
prosperidad futura» (traducción de Bádenas-Bernabé 1984). Es difícil reconocer el final 
del proceso en el sentido del preverbio en este caso, en aparente contradicción con el uso del presente. Probablemente acierta el comentario de Gentili et alii (1995, p. 504) que lo entiende como equivalente a un perfecto («sono preparati, scil. dagli dei, cfr. Sch.489d $\pi \alpha \rho \alpha \grave{\alpha} \tau \tilde{\omega} \nu \theta \varepsilon \tilde{\omega} \nu \kappa \alpha \tau \alpha \sigma \kappa \varepsilon \cup \alpha ́ \zeta \zeta o v \tau \alpha(»)$ y considera que el presente durativo «sottolinea que Arcesilao non ha ancora questo favore (anche sé, il poeta ne è certo, lo avrà in futuro»).

5. غ̇лณков́ஸ escuchar, atender en cont. de súplica (Pi.O.14.15)

Este lema no aparece en LSJ, que incluye esta cita de Píndaro en el artículo غ̇пท́коo, dentro de los usos referidos a dioses, advirtiendo de que es la lectura de los códices. Efectivamente, la edición de Snell-Maehler, como otras anteriores, acepta la



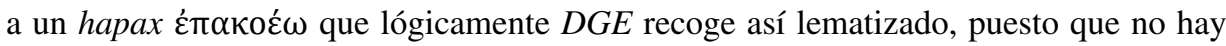
documentada forma jónico-ática equivalente. Sin embargo, al parecernos preferible, como a LSJ y a otros muchos autores, la lectura de los códices y del escolio, añadimos información textual y remitimos al artículo غ̇пท́коо , donde también se mantiene esta cita señalando que es la lectura de los códices:

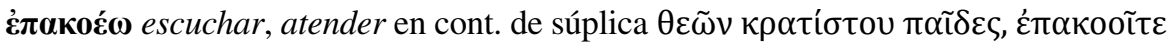

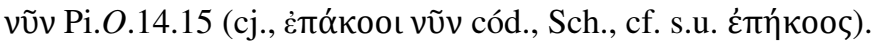

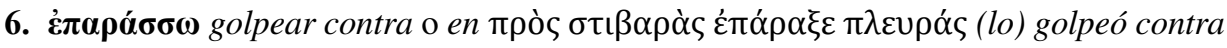
sus fuertes costillas Heracles a Anteo (Pi.Fr.111.4)

Esta cita constituye un addendum a LSJ procedente de la edición de Maehler de este fragmento transmitido por Erotiano. La variante $\dot{\pi} \pi \alpha ́ \rho \alpha \xi \varepsilon$ resulta claramente preferible a las demás ${ }^{3}$ y aporta, además de la más antigua documentación del compuesto, una construcción nueva, pues tenemos diversas construcciones del verbo para expresar lo gol-

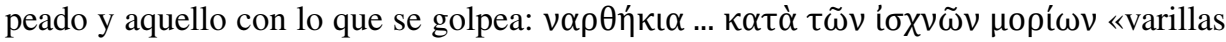

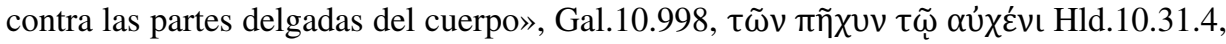

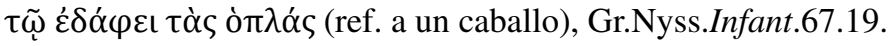

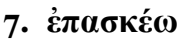

a) fig. añadir adorno al vencedor o a su ciudad, e.d. exaltar también (Pi.N.9.10, Fr.194.4)

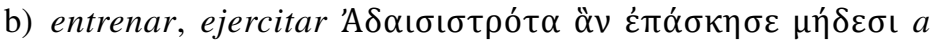
la que entrenó Adesístrota con sus consejos en la danza (Pi. Fr.94b.71).

En LSJ estas tres citas pindáricas aparecen reunidas en un apartado exclusivo para

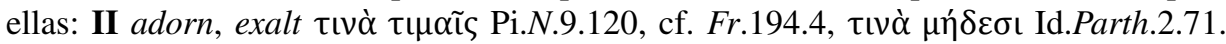

\footnotetext{
${ }^{3}$ Cf. el comentario de Lobel a su edición del POxy.2446 fr.1, que coincide parcialmente con el texto transmitido por Erotiano.
} 
De una lectura atenta de los restos del segundo partenio, que ahora citamos Fr.94b.71, resulta evidente que la tercera cita debe ir incluida en el apartado en el que el verbo, con acusativo de persona, significa entrenar, ejercitar (DGE s.u. II 2). En este apartado encontramos citas muy posteriores referidas al deporte (IG $2^{2} .3015 .8$, del III d.C.) y al entrenamiento militar (Arr.Tact.1.6.1), pero también a saberes como la retórica (Hermipp. Hist.33), o la medicina (Gal.1.62).

Quedan los otros dos pasajes como únicos representantes de este empleo figurado en el que el poeta o el poema adornan, es decir exaltan u honran al vencedor: N.9.10 $\tilde{\omega} \mathrm{v}$

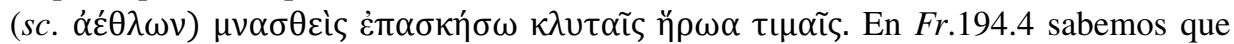

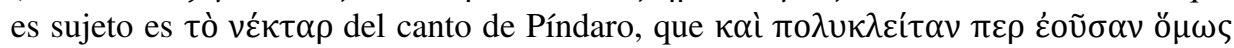

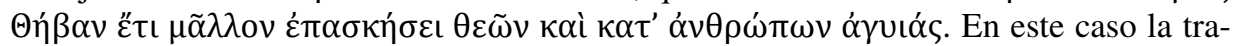

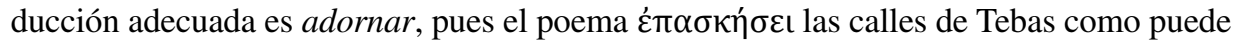
hacerlo una construcción arquitectónica o una estatua.

Pero lo principal es que hasta ahora no se ha reparado, como en tantas otras ocasiones, en el valor del preverbio. Aquí ć一( ( )- presenta uno de sus más frecuentes valores en composición, cuya noción básica es en añadidura. A partir de aquí hay distintas traducciones posibles según los casos. Muy frecuentemente lo relevante es que aquello que se añade o la acción que viene a añadirse lo hace con posterioridad, por lo que el preverbio adquirirá valor temporal y traduciremos a continuación, después. Pero cuando no hay esta sucesión temporal muy frecuentemente traduciremos además, también y, si se trata de acciones verbales, a menudo la traducción adecuada es a la vez. En N.9-10 no se trata

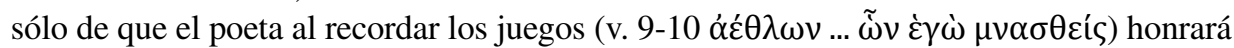
o exaltará al héroe con gloriosos honores, sino que lo hará a la vez, es decir, el recuerdo de los juegos en el canto servirá también para exaltar al héroe.

Este sentido del preverbio aparece claramente en los más antiguos usos del compuesto, en los que se construye con un acusativo de resultado y significa construir con

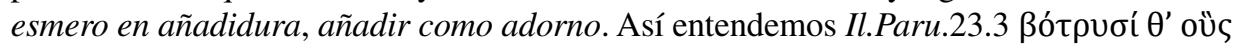

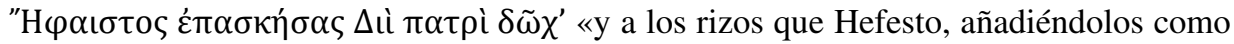
adorno, dió a Zeus padre» y sobre todo podemos entender el uso pasivo de Od.17.266,

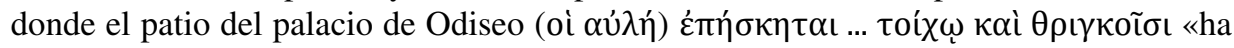
sido adornado añadiéndole muro y cornisa», es decir, «se le han añadido muro y cornisa como adorno».

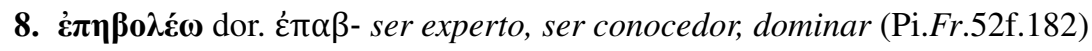

En el primer suplemento a LSJ (LSJ Suppl. 1968) aparecía por primera vez recogido este lema, a partir de la documentación en un fragmento yámbico anónimo, quizá procedente de la obra de Arquíloco, y en el penúltimo verso del sexto Peán de Píndaro. Allí también se ofrecía la traducción gain, win, añadiendo que en Píndaro se construía con genitivo y en el adespoton yámbico con acusativo. El segundo suplemento a LSJ (LSJ Rev.Suppl. 1996) de Glare mantenía la misma documentación, cambiando la traducción a «achieve mastery, knowledge, etc. of». Glare podría haber señalado el tercer testimo-

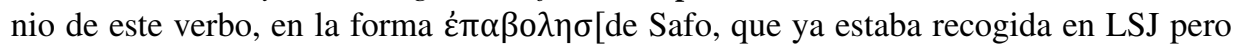
s.u. غ̇ंท́ $\beta$ o $\lambda$ os, procedente del suplemento a la edición de los líricos de Diehl (Sapph. Supp. $10.2=21.2$ Voigt). 
9. غ̇ंі́крvфоธ que se hace a escondidas (Pi.O.8.69)

Píndaro está hablando de los derrotados en los juegos que, al contrario que el vence-

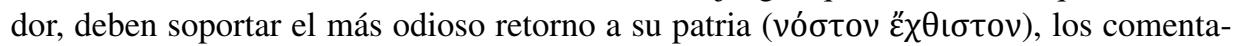



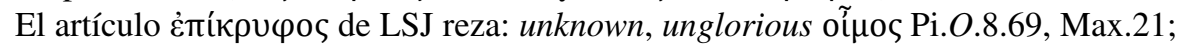

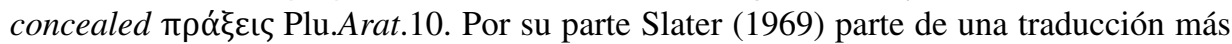
literal, de acuerdo con la etimología del término, para llegar a la misma interpretación: hidden ..., i.e. inglorious. En realidad, uno y otro van más allá de lo que dice el griego.

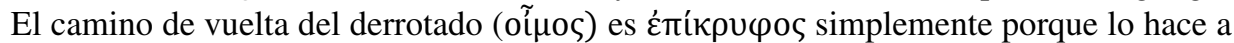
escondidas, ocultándose él mismo de los demás. Identificamos así un solo significado y el mismo en las citas de Píndaro y Máximo de un lado y en la de Plutarco, de otro, donde

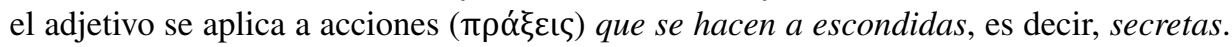
Los comentarios y los traductores de Píndaro suelen entenderlo así. Por ejemplo Suárez («y el camino a escondidas»); algo más libremente, recogen la misma idea Ortega («la entrada por encubierto paso») y Bádenas-Bernabé («y el caminar a hurtadillas»).

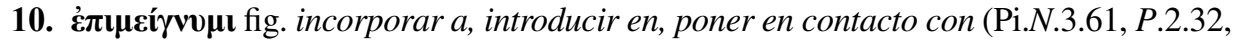
N.3.62, Fr.94b.5)

Verbo favorito de Píndaro, que lo emplea siempre en un uso figurado difícil, por lo que no siempre ha sido bien entendido. Basta ver la redacción del apartado transitivo (I) del artículo émı $\mu \varepsilon^{\prime} \gamma v u \mu$ de LSJ, donde, tras un único uso propio del verbo en Nicandro, recoge las cuatro citas de Píndaro con diferentes traducciones no muy coherentes entre sí, más una de Platón que, hasta el momento, sigue constituyendo el único paralelo del uso pindárico:

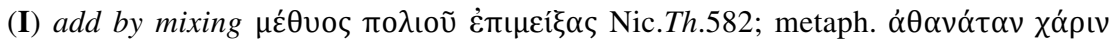

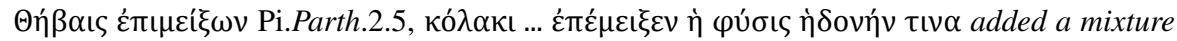

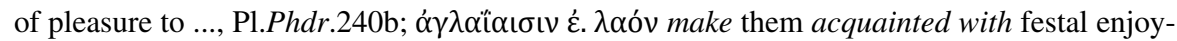

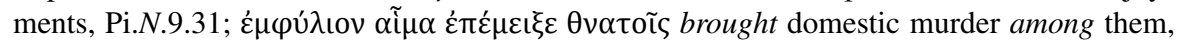

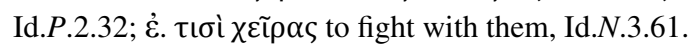

$D G E$, que aumenta considerablemente el número de citas transitivas con respecto a LSJ, tiene un subapartado I 1 para los usos propios, donde efectivamente el verbo significa añadir mezclando, pero ponemos a continuación la traducción más adecuada al español, que también nos va a dar la clave de los usos figurados: incorporar. Así, alguien incorpora una sustancia a otra, es decir, añade una substancia a otra mezclándola con ella:

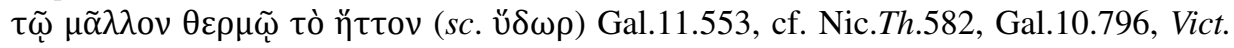

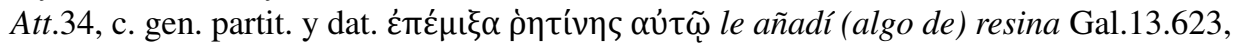
cf. Paul.Aeg.3.48.4.

Del mismo modo, en los usos figurados, donde las substancias concretas son sustituidas por abstractos, se trata de incorporar por primera vez algo a alguien (dat. de pers.) o de incorporar a alguien (ac. de pers.) a algo, según el complemento de persona aparezca en dativo y el abstracto en acusativo, lo más frecuente, o a la inversa. Se trata de poner dos elementos — persona y abstracto- en contacto por primera vez, y en 
ocasiones podemos para ello utilizar la traducción incorporar. Pero ya no se trata del mismo significado en un uso metafórico eventual, se trata de dos significados diferentes,

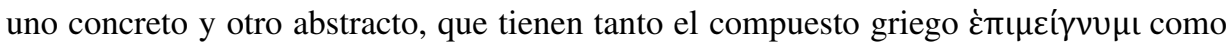
el español incorporar. Por eso los usos abstractos aparecen en un subapartado especial (I 3) donde se añaden otras traducciones alternativas — que nunca podrían utilizarse para traducir los usos propios del compuesto- y que resultan más adecuadas según los

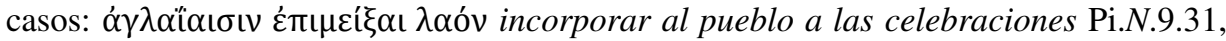

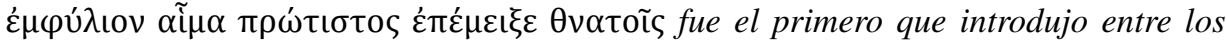

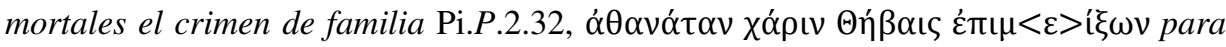
introducir (Apolo) su gracia inmortal en Tebas Pi.Fr.94b.5. Algo especial resulta el

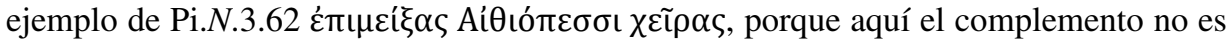
abstracto sino concreto. Aunque el significado es básicamente el mismo, resulta preferible ofrecer dos traducciones, una literal y otra más libre que permita entender el pasaje en su conjunto: poniendo sus manos en contacto con los etiopes, e.d. trabando combate con los etíopes Pi.N.3.62.

\section{BIBLIOGRAFÍA}

D’ Alessio, Gian Battista, - Ferrari, Franco (i988). «Pindaro, Peana 6, 175-183: una ricostruzione», SCO 38, 159-179.

Bádenas, Pedro - Bernabé, Alberto (i984). Píndaro, Epinicios. Edición de P. Bádenas de la Peña y A. Bernabé. Madrid, Alianza (Madrid, Akal, 2002²).

Gentili, Bruno et alii (I995). Pindaro, Le Pitiche. A cura di B. Gentili, P.A. Bernardini, E. Cingano e P. Giannini. [Verona], Fondazione Lorenzo Valla - Mondadori.

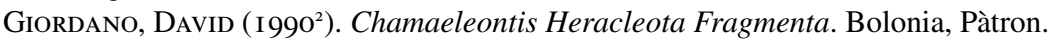

LenNartZ, KARL (2000). «Fragmentum iambicum adespotum 38 W.», Philologus 144, 2000, 22-29.

LSJ: A Greek English Lexicon. Compiled by H.G. Liddell and R. Scott, revised and augmented throughout by H.S. Jones. 9th. Edition (1940). Oxford, Clarendon Press.

LSJ: REv.Suppl. (1996). Greek-English Lexicon. Revised Supplement. Edited by P.G.W. Glare and A.A. Thompson. Oxford, Clarendon Press.

Maehler, Herwig (i989). Pindari Carmina. Pars II. Fragmenta. Indices. Leipzig, Teubner.

Ortega, Alfonso (I984). Píndaro, Odas y fragmentos. Introducciones, traducción y notas de A. Ortega. Madrid, Gredos.

Privitera, G. Aurelio (1982). «Due note alla prima Istmica di Pindaro», en E. Livrea - G.A. Privitera (eds.), Studi in Onore di Anthos Ardizzoni, Roma, Edizioni dell' Ateneo e Bizzarri, pp. 723-734.

RUTHERFORD, IAN (200I). Pindar's Paeans. A reading of the fragments with a survey of the genre. Oxford, Oxford University Press.

Slater, William J. (1969). A Lexicon to Pindar. Berlín, De Gruyter. Snell, Bruno - Maehler, Herwig (1987). Pindari Carmina. Pars I. Epinicia. Post B. Snell edidit H. Maehler. Leipzig, Teubner.

SuÁrez, Emilio (i988). Píndaro, Obra completa. Edición de E. Suárez de la Torre. Madrid, Cátedra. 
